\title{
Syphilis serology: Seroprevalence in a selected population and considerations on the Euroline WB test
}

\author{
Andrea Amodeo, Francesco Caccamo, Emanuela Fichera, Patrizia Grassi, Elena Grasso, Giuliana Guardo, \\ Silvana Mastrojeni, Ildebrando Patamia, Vanessa Scriffignano, Agata Sciacca \\ Laboratory Analysis Unit Policlinico, University of Catania, Italy.
}

Key words: Syphilis, seroprevalence, western blot

Sierologia della Sifilide: sieroprevalenza in una popolazione selezionata e considerazioni metodologiche sul test eurolineWB

\section{SUMMARY}

Introduction: The clinical diagnosis of syphilis is always supported by appropriate laboratory tests and the test results are interpreted with reference to the patient's history.

In the diagnosis of syphilis, the use of tests based on antibody search that recognize both treponemal and reaginic antigens increases the diagnostic chances.

Our study discusses the various serological and alternative tests currently available along with their limitations, and relates their results to the likely corresponding clinical stage of the disease.

Methods: in our laboratory were analyzed 264 sera and 4 liquor (I23 Females, I45 Males).

187 patients are subject at low risk for luetic infection, including pregnant woman, patient with organ transplant, outpatients or hospitalized undergoing routine serological, and 81 from patients with confirmed syphilis including 4 pregnant women in antibiotic treatment, patients with suspected disease, HIV positive and patients with autoimmune diseases with Cardiolipin positive.

All sera were tested with ELISA Anti-Treponema pallidum Screen (IgG / IgM) and in parallel with agglutination tests VDRL and TPHA. On all positive sera was tested Euroline-WB EUROIMMUN and reading done with the program EuroLineScan.

Results: by ELISA Anti-Treponema pallidum Screen IgG / IgM I62 sera were negative and I06 sera positive (39.5\%), distributed as follows: 45 (42\%) with a value greater than $200 \mathrm{RU} / \mathrm{ml}, 43$ (4I\%) with a value $>22 \mathrm{RU} / \mathrm{ml}$ and I8 (I7\%) with a borderline value between $>$ I6 to <22 $\mathrm{RU} / \mathrm{ml}$. The execution of the Blot lgG showed: I 8 negative sera, 6 with borderline value with one only band of specific antigens ( $\mathrm{p} / 5$, $\mathrm{p} 45$, p47 or p 17), while 82, including 4 liquor (neurolue), were certainly positive showing more than one band antibody to the treponemal antigens. Only one patient had in place at the time of screening, an initial infection; in fact, there was a single clear positivity in the IgM protein bands, while 7 sera was uncertain values. It is reported II positivity for IgM Cardiolipin, while Cardiolipin IgG was detected with a high positivity in 34 sera.

The presence of borderline values and / or positivity for a single protein band can be attributed to a unspecific reaction caused by autoimmune diseases or related cross-reactions with other Spirochete or to other Borrelia.

Conclusions: The immunoblot test gave useful information at epidemiological and clinical level.

The deepening with a confirmation test with proteic antigens and cardiolipin identifies false reactivity, but also indicates the specific reactivity to past infection and a better characteriation in the different stages of disease. In our study in the latent forms there are relevant discrepancies among the various tests. Compare to traditional methods, anti-cardiolipin antibodies positivity in our confirmatory test has the advantage of providing non subjective interpretation, being based upon the EuroLineScan program.

\section{INTRODUZIONE}

La diagnosi della sifilide primaria si basa su dati clinici, ma nelle forme secondarie tardive e ancor più nelle forme latenti, l’impiego dei test sierologici è fondamentale.

Quando il paziente presenta delle lesioni, i mezzi migliori per la diagnosi di lue sono l'esame in campo oscuro e l'immunofluorescenza diretta (DFA-TP: Direct Fluorescent AntibodyTreponema pallidum testing).

I test sierologici sono di ausilio alla diagnosi di lue primaria e secondaria rispetto al DFA-TP, ma sono gli unici metodi per la diagnosi della sifilide latente precoce e sifilide tardiva.

Essi sono divisibili in due categorie: treponemici e non treponemici (o reaginici). I reaginici sono utili per fornire un'indicazione sull'attività della malattia (4).

Gli anticorpi prodotti come risposta all'infezione di Treponema pallidum sono dosabili già durante lo stadio primario (1-4 settimane dopo la comparsa del sifiloma) e aumentano nella sifilide secondaria.

I titoli reaginici diminuiscono dopo la terapia, diventando negativi nell'arco di 1 anno nella sifilide primaria e di 2 anni nei casi secondari. I test reaginici non diventano positivi prima di 3-6 settimane dall’infezione iniziale. Poiché il sifiloma primario si manifesta prima di tale epoca, un test sierologico precoce negativo non può escludere la sifilide. Nei pazienti con lesioni genitali, i test reaginici devono dare risultati non reattivi alla sesta settimana prima che si possa esclu- dere la diagnosi di sifilide (2).

I test treponemici solitamente divengono positivi entro 3-4 settimane e restano tali per molti anni, nonostante il paziente abbia effettuato una terapia efficace.

L’impiego di test basati sulla ricerca nel paziente di anticorpi che riconoscono sia antigeni treponemici $(1,3,5)$ che reaginici aumenta le possibilità diagnostiche.

\section{MATERIALI E METODI}

Presso il nostro laboratorio sono stati analizzati 264 sieri e 4 liquor (123 Femmine e 145 Maschi): 187 sieri provenivano da soggetti a basso rischio per infezione luetica, di questi 40 appartenevano a donne in gravidanza, 20 a pazienti sottoposti o candidati a trapianti d'organo e 127 a pazienti esterni o ospedalizzati sottoposti ad analisi sierologiche di routine.

I restanti 81 erano di pazienti con sifilide accertata tra cui 4 donne in gravidanza in trattamento antibiotico, 70 con sospetto di malattia luetica e 7 HIV positivi.

Tutti i sieri sono stati analizzati con test ELISA AntiTreponema pallidum Screen (IgG/IgM) e in parallelo con test di agglutinazione VDRL e TPHA. Tutti i sieri positivi sono stati testati tramite Euroline-WB EUROIMMUN e la lettura effettuata con il programma EuroLineScan.

Il test kit EUROLINE WB è un test qualitativo in vitro per la determinazione degli anticorpi umani di classe IgG/IgM, presenti nel siero o nel plasma, contro il Treponema pallidum. 
Il test kit contiene strip coattate con estratti antigenici di Treponema pallidum, separati mediante elettroforesi. Le proteine principali specifiche per Treponema pallidum sono quelle con peso molecolare di 47- 45.5 -17 e $15.5 \mathrm{kDa}$. (Tabella 1). Inoltre, ogni strip possiede un chip di membrana coattato con la cardiolipina a tre concentrazioni diverse.

Inizialmente, le strip vengono pre-trattate ed incubate con i campioni di siero o di plasma diluiti.

Se il campione è positivo, anticorpi specifici di classe IgG/IgM si legheranno all'antigene corrispondente per evidenziare gli anticorpi legati. Si segue una seconda incubazione utilizzando anticorpi anti-IgG umane coniugati con un enzima (coniugato enzimatico) in grado di promuovere una reazione colorimetrica. La lettura delle strip viene effettuata automaticamente utilizzando EuroLineScan.

I risultati del test EUROLINE WB per gli anticorpi antiTreponema pallidum possono essere suddivisi in negativi, positivi e borderline (Tabella 2).

Per valutare i risultati, è necessario tenere in considerazione sia la posizione delle bande sia la loro intensità; a volte, infatti, campioni negativi possono determinare una debole colorazione a livello di singole bande antigeniche.

Lo screening in ELISA consiste in un test immunoenzimatico semiquantitativo o quantitativo per la ricerca degli anticorpi di classe IgG e IgM specifici verso il Treponema pallidum.

Vengono considerati positivi per la presenza di anticorpi antiTreponema pallidum i campioni con valore >22 RU/ml; negativi se $<16$ e dubbi quelli tra 16 e 22.

\section{RISULTATI}

Con il test ELISA anti-Treponema pallidum Screen IgG/ IgM 162 sieri sono risultati negativi e 106 sieri positivi (39.5\%), così distribuiti:

- 45(42\%) con un valore superiore a $200 \mathrm{RU} / \mathrm{ml}$,

- 43(41\%) con un valore compreso fra 22 e $200 \mathrm{RU} / \mathrm{ml}$

- 18(17\%) con un valore borderline compreso fra 16 e 22 $\mathrm{RU} / \mathrm{ml}$.

I risultati ai test ELISA, VDRL, TPHA, Euroline-WB IgG e

Tabella I. Specificità degli antigeni presenti sulle strip Euroline-WB

\begin{tabular}{lll}
\hline Banda & Antigene & Specificità \\
\hline $47 \mathrm{kDa}$ & Proteina di membrana,TpN47 & Specifica \\
\hline $45 \mathrm{kDa}$ & TmpA & Specifica \\
\hline $\mathbf{2 2} \mathrm{kDa}$ & P22 & Aspecifica \\
\hline $17 \mathrm{kDa}$ & Proteina di membrana,TpNI7 & Specifica \\
\hline $\mathbf{1 5} \mathrm{kDa}$ & Proteina di membrana,TpN I5 & Specifica \\
\hline $\mathrm{CL} \mathrm{3}$ & Cardiolipina & Marcatore di attività, aspecifica \\
\hline $\mathrm{CL} \mathrm{2}$ & Cardiolipina & Marcatore di attività, aspecifica \\
\hline $\mathrm{CL} \mathrm{I}$ & Cardiolipina & Marcatore di attività, aspecifica \\
\hline
\end{tabular}

Tabella 2. Interpretazione dei risultati al test Euroline-WB

\begin{tabular}{ll}
\hline Risultato & Caratteristiche \\
\hline Negativo & Nessuna banda a livello di specifici antigene \\
\hline Borderline & Una banda distinta dello specifico antigene: p I5 kDa, pI7 kDa, p45 kDa o p47 kDa \\
\hline Positivo & Più di una banda distinta dello specifico antigene: p I5 kDa, p I7 kDa, p45 kDa o p47 kDa \\
\hline
\end{tabular}

Tabella 3. Risultati ai test ELISA,VDRL,TPHA, Euroline-WB $\lg G$ e $\lg M$ messi a confronto

\begin{tabular}{|c|c|c|c|c|}
\hline \multicolumn{5}{|c|}{ Risultati dei vari test messi a confronto } \\
\hline ELISA & VDRL & TPHA & EUROLINE-WB IgG & EUROLINE-WB IgM \\
\hline & $\begin{array}{l}\text { 6I positivi } \\
>1: 2\end{array}$ & $\begin{array}{l}66 \text { positivi }>1 / 80 \\
<1 / 80\end{array}$ & $\begin{array}{l}82 \text { positivi } \\
\text { (positivi a più antigeni proteici) }\end{array}$ & I (p|7a p45 p47) \\
\hline \multirow[t]{4}{*}{ I06 positivi } & & & $\begin{array}{l}6 \text { borderline } \\
\text { (positivi ad un solo antigene) }\end{array}$ & \\
\hline & $\begin{array}{l}44 \text { negativi } \\
<1: 2\end{array}$ & $\begin{array}{l}39 \text { negativi } \\
<1 / 80\end{array}$ & I8 negativi & \\
\hline & & & 34 positivi & II positivi \\
\hline & & & Cardiolipina IgG & Cardiolipina IgM \\
\hline 162 negativi & I62 negativi & I62 negativi & & \\
\hline
\end{tabular}

IgM sono riportati in Tabella 3.

L'esecuzione del Blot IgG ha evidenziato:18 sieri negativi, 6 con un valore borderline con una sola banda degli antigeni specifici e 82 (tra cui i 4 liquor ) sono risultati sicuramente positivi presentando più di una banda anticorpale agli antigeni treponemici (Tabella 3, Figura I).

Solo un paziente presentava in atto, al momento dello screening, una prima infezione; infatti, è stata riscontrata una sola positività nelle bande proteiche IgM, mentre 7 sier presentavano valori dubbi. Si segnalano 11 positività per Cardiolipina IgG .

La presenza di valori borderline e/o la positività per una sola puo essere attribuita ad una reazione aspecifi-

\section{DISCUSSIONE}

Anche se la sifilide è una malattia antica e conosciuta, l'attuale andamento dell'infezione si configura come un fenomeno molto complesso che pone una grande sfida, sia a livello diagnostico sia terapeutico, finalizzato al controllo dell'infezione a livello mondiale . L'eliminazione di questa malattia è considerata un obiettivo biologicamente ed epidemiologicamente raggiungibile in quanto non esistono reservoir animali e si può diagnosticare e curare con farmaci a basso costo. Nuove luci sulla patogenesi stanno arrivando dall'analisi del genoma batterico e permetteranno di elaborare nuove strategie per il trattamento, il controllo e lo sviluppo dei vaccini. Il test immunoenzimatico ha dato delle informazioni utili a livello epidemiologico e clinico e infatti è stato in grado di identificare sia le infezioni luetiche recenti che le forme latenti e le pregresse infezioni trattate e superate. La prevalenza di anticorpi (14\%) anti Treponema pallidum nella popolazione a basso rischio analizzata, suggerisce che vi sia una maggiore diffusione dell'infezione nella popolazione generale, da attribuire sicuramente a una variazione delle abitudini sessuali, a una maggiore promiscuità, ma soprattutto a una minore percezione del rischio di infezioni trasmissibili. Un risultato positivo con un test in ELISA sensibile deve stimolare ulteriori indagini.

L'approfondimento con un test di conferma con antigeni proteici e cardiolipina individua le false reattività e le possibili interferenze in una popolazione, ma soprattutto indica la reattività specifica a un'infezione pregressa e consente quindi una migliore caratterizzazione nelle 
varie fasi della malattia. La positività ai test VDRL e RPR indica una diagnosi di sifilide primaria o trattata di recente o non trattata. La positività degli anticorpi anti-cardiolipina nel test di conferma da noi usato ha il vantaggio, rispetto ai metodi tradizionali, di una interpretazione non soggettiva, ma elaborata con il programma Eurolinescan.

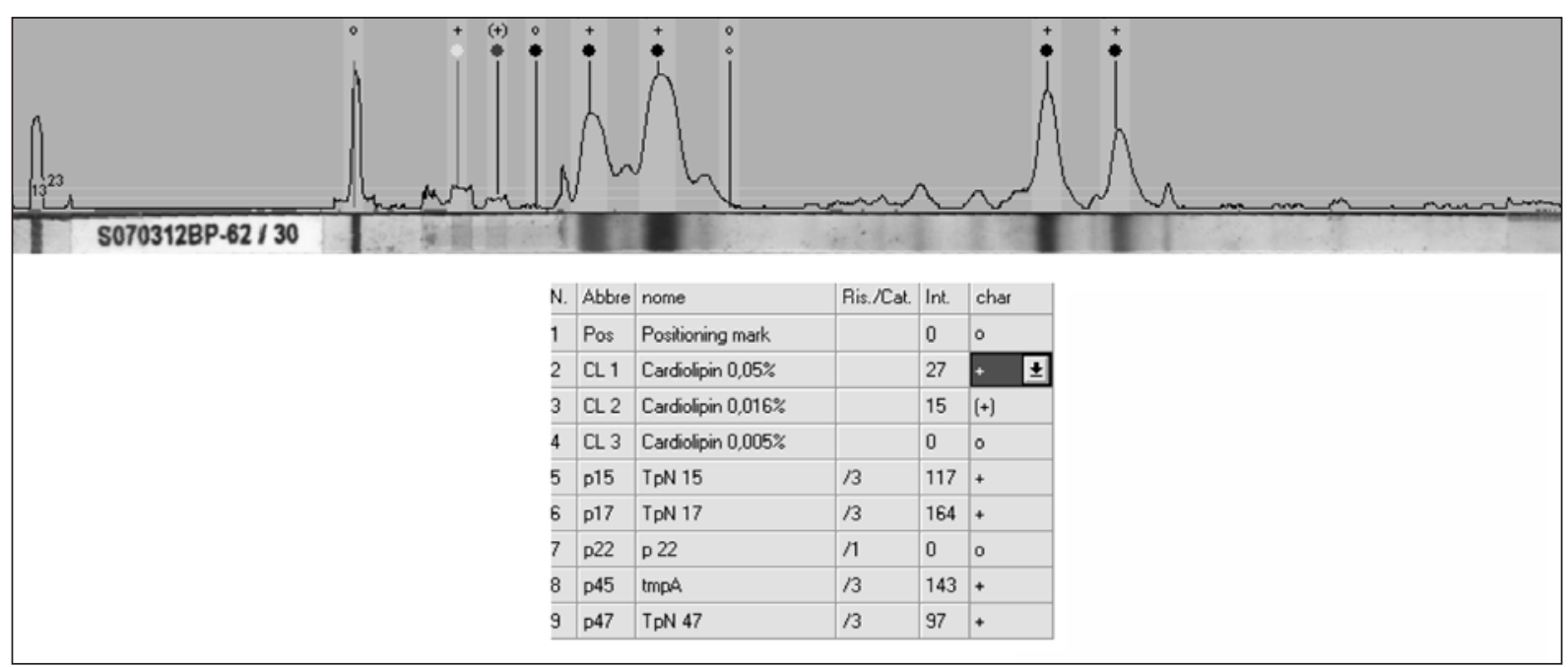

Figura I. Esempio di lettura di una strip del test Euroimmun-WB elaborata con il programma Eurolinescan: donna in gravidanza positiva alle lgG.

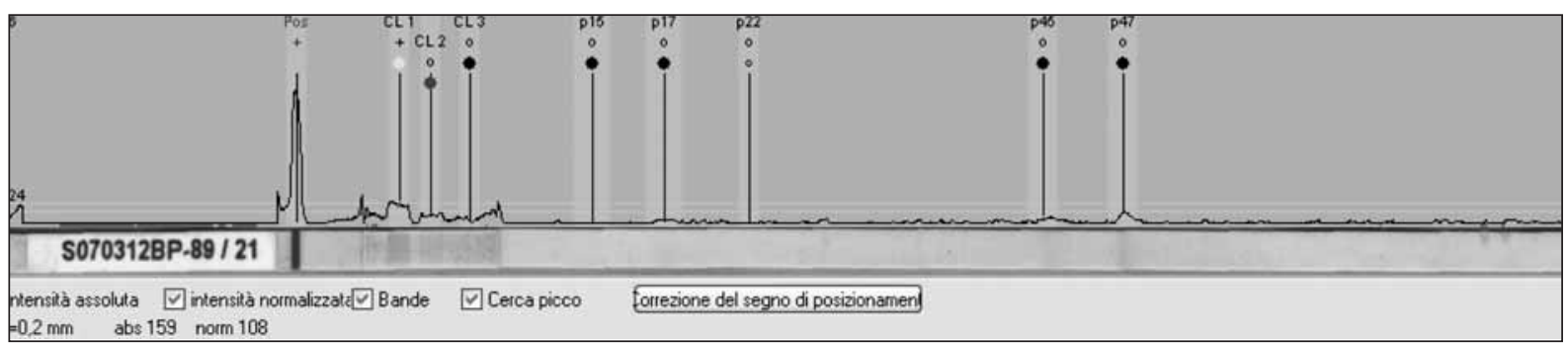

Figura II. Esempio di lettura di una strip del test Euroimmun-WB elaborata con il programma Eurolinescan: IgM positiva: interferenza con malattie autoimmuni.

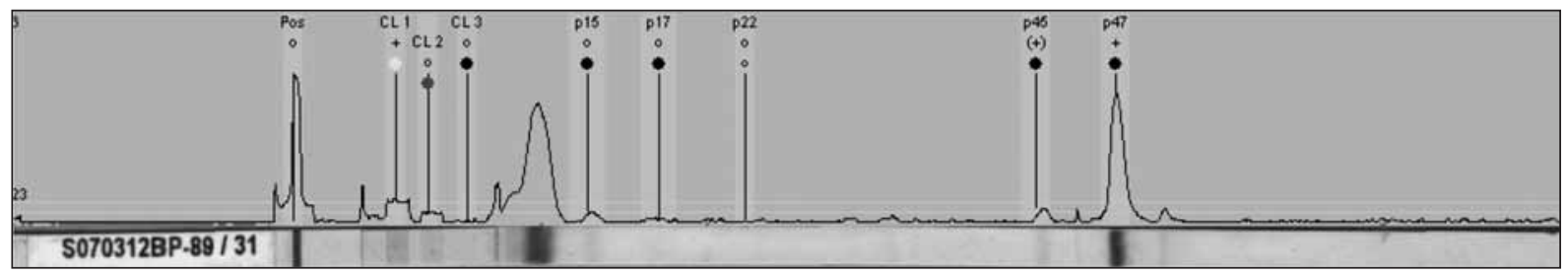

Figura III. Esempio di lettura di una strip del test Euroimmun-WB elaborata con il programma Eurolinescan: IgG positiva: interferenza con malattie autoimmuni.

\section{BIBLIOGRAFIA}

1. Codd AA, Sprott MS, Narang HK, et al. Competitive enzyme-linked immunosorbent assay for Treponema pallidum antibodies. J Med Microbiol 1988; 26:153.

2. Giacani L, Sambri V, Marangoni A, et al. Immunological evaluation and cellular location analysis of TprI antigen of Treponema pallidum subs. pallidum. Infect Immun 2005; 73: 3817-3822.

3. La Placa M, Barbanti Brodano G, Bendinelli M et al. Introduzione allo stu- dio della microbiologia medica. In: Principi di Microbiologia Medica. Bologna: Società Editrice Esculapio, 1995: 1-32.

4. La Placa M, Barbanti Brodano G, Bendinelli $\mathrm{M}$ et al. Spirochete. In: Principi di Microbiologia Medica. Bologna: Società Editrice Esculapio,1995: 351-357.

5. Lukehart SA. Identification and characterization of Treponema pallidum antigens by monoclonal antibodies. In: Monoclonal antibodies. Academic Press, 1986:1. 